\title{
Algunas expectaciones acerca del asma
}

\author{
Some expectations about asthma
}

\section{Algumas expectativas sobre a asma}

Vanessa M. Luzardo-Palacios II

vaneluzardo@hotmail.com

Vanessa M. Cedeño-Zavalu III

vane-ceza@hotmail.com

Recibido: 10 de septiembre de 2017 * Corregido: 10 de noviembre de 2017 * Aceptado: 28 de diciembre de 2017

I. Doctora en Medicina y Cirugía, Médico Especialista en Neumonologia, Docente de la Universidad Laica Eloy Alfaro de Manabí, Manta, Ecuador.

II. Doctor en Medicina y Cirugía, Médico Especialista en Alergia e Inmunología, Docente de la Universidad Laica Eloy Alfaro de Manabí, Manta, Ecuador. 


\title{
Resumen
}

El asma es una enfermedad respiratoria crónica que implica la inflamación y estrecharse de las aerovías que sea una de las enfermedades no-transmisibles mayores por todo el mundo. A tales efectos se realizó una revisión bibliográfica, exhaustiva para exponer algunos aspectos relacionados con esta afección (definición, patogenia, factores de riesgo, aspectos vitales para el diagnóstico, entre otros. El conocimiento de esta enfermedad, llevará a una mejor evolución de los pacientes y un abordaje global de los síntomas y la aparición de crisis.

Palabras clave: asma; enfermedad crónica no trasmisible.

\begin{abstract}
Asthma is a chronic respiratory disease that involves inflammation and narrowing of the airways that is one of the major noncommunicable diseases worldwide. To this end, a bibliographic review was carried out, exhaustive to expose some aspects related to this condition (definition, pathogenesis, risk factors, vital aspects for diagnosis, etc.) Knowledge of this disease will lead to a better evolution of patients and a global approach to symptoms and the appearance of crises.
\end{abstract}

Keywords: asthma; chronic non-communicable disease

\section{Resumo}

A asma é uma doença respiratória crônica que envolve inflamação e estreitamento das vias aéreas, que é uma das principais doenças não-transmissíveis em todo o mundo. Para este fim, foi realizada uma revisão bibliográfica, exaustiva para expor alguns aspectos relacionados a esta condição (definição, patogênese, fatores de risco, aspectos vitais para o diagnóstico, etc.) O conhecimento dessa doença levará a uma melhor evolução dos pacientes e uma abordagem global dos sintomas e a aparência de crises.

Palavras chave: asma doença crônica não transmissível

\section{Introducción}

El asma es la enfermedad crónica más común de la infancia; representa la primera causa de ausentismo escolar, consultas urgentes al pediatra o al servicio de urgencias y hospitalización. ${ }^{1}$ 
El impacto de esta enfermedad es tremendo, no solo por su alta morbilidad, sino por el efecto económico y social asociado a ella. Frecuentemente, la enfermedad y los síntomas nocturnos acompañados con las alteraciones del sueño, tanto en los niños como en sus padres, dan como resultado un desempeño escolar pobre y días de trabajo perdidos. ${ }^{1}$

Actualmente se considera que las principales causas que contribuyen a la morbimortalidad del asma son el subdiagnóstico y el manejo inadecuado; factores que muy probablemente sean la causa de una educación médica inadecuada. Nuestro objetivo en la presente revisión es mostrar las principales evidencias científicas para su diagnóstico y manejo de este padecimiento. ${ }^{1}$

\section{Definición}

El concepto de asma ha sido evolutivo. Hasta 1975 se consideraba que el asma se producía por una contracción de los bronquios; con el advenimiento de la fibrobroncoscopía y el lavado broncoalveolar se descubrió que además existe una inflamación crónica de la vía aérea, que genera la sintomatología; posteriormente, el progreso en las técnicas de biopsia bronquial llevó a reconocer que lo que se creía en la década de 1990, en el sentido de que el asma es una enfermedad reversible, no era exacto, ya que algunos pacientes desarrollaban algún grado de fibrosis y remodelación de la vía aérea secundarias a la inflamación crónica repetitiva, lo que explica que con el tiempo, el paciente asmático no controlado presente obstrucción persistente. ${ }^{2}$

Debido a su etiología multifactorial, a las alteraciones funcionales respiratorias y a sus diversas expresiones clínicas, de acuerdo a la edad de presentación, no hay una definición que describa completamente a esta entidad. Por lo tanto, definir asma es complejo, ya que es una enfermedad multifactorial (etiología, características morfológicas, cambios funcionales y manifestaciones clínicas), en la cual existe una interacción entre el sistema inmunológico y el sistema nervioso autónomo. Sin embargo, la definición más completa es la que toma en cuenta tanto el punto de vista funcional como el celular, es por eso que la definición que mejor se adapta es la propuesta por la Iniciativa Global para el Asma (GINA 2006), ${ }^{3}$ que propone al asma como una enfermedad inflamatoria crónica de las vías aéreas, en la cual muchas células y productos celulares juegan un papel importante. La inflamación crónica produce un incremento en la reactividad de la vía aérea y 
episodios recurrentes de sibilancias, dificultad respiratoria, tiraje intercostal, tos y opresión torácica, especialmente en la noche y en la mañana.

La Guía de manejo y prevención del asma en adultos y niños mayores de 5 años GINA 2015, señala que:

El asma es una enfermedad heterogénea, generalmente caracterizada por la inflamación crónica de las vías respiratorias. Se define por la historia de los síntomas respiratorios como sibilancias, dificultad para respirar, opresión en el pecho y tos que varían en el tiempo y en intensidad, junto con la limitación del flujo aéreo espiratorio variables. ${ }^{4}$

\section{Epidemiología}

El asma bronquial es una enfermedad de alta prevalencia, que causa morbilidad y mortalidad. En las dos últimas décadas se ha visto un incremento importante de esta patología, que ha llegado a ser una de las enfermedades crónicas más comunes en el mundo, en especial en los países desarrollados y en la población infantil, ha ido aumentando en gravedad y letalidad, es decir, en la actualidad hay más asmáticos, más asmáticos graves y estos últimos se mueren más que antes. Además, la enfermedad provoca elevados gastos en salud, ya que en muchos casos el tratamiento adecuado del paciente asmático se debe mantener de por vida; así, en países desarrollados, como España, se gasta $1 \%$ a $2 \%$ del presupuesto de salud en programas de asma. Se proyecta que los países en vías de desarrollo, como Chile, tendrán un incremento de las consultas por asma, de modo que es importante considerar que el asma no controlada es costosa y que la inversión en fármacos preventivos podría disminuir los costos en atención.

La incidencia de asma va en aumento en todo en el mundo. Algunos datos señalan que la incidencia llegó a sus niveles más altos entre 1960 y 2000, en especial en los países en vías de desarrollo, que posteriormente se ha estabilizado y que este aumento ocurrió más en áreas urbanas que rurales. ${ }^{2}$

Según Lang J. ${ }^{5}$ el asma es una de las enfermedades crónicas más comunes que se presenta en los niños, la cifra de personas en el mundo afectadas puede ser tan alta como alrededor de 300 millones; el asma constituye una carga en la calidad de vida y los costes sanitarios en Estados Unidos y es la causa principal de hospitalización, visitas a urgencias y ausencia a la escuela. 
En el Ecuador, se estima que el asma afecta al 7\% de la población. De acuerdo con las cifras del Instituto Ecuatoriano de Estadística y Censos (INEC), en el 2010 se registraron en el país 3275 casos de esta enfermedad. ${ }^{6}$

De acuerdo con las cifras del Instituto Ecuatoriano de Estadística y Censos (INEC), en el 2010 se registraron en el país 3275 casos de esta enfermedad. ${ }^{6}$

\section{Patogenia}

No todos los niños que presentan síntomas de asma van a llegar a ser adolescentes asmáticos, ni todos estos persistirán con esta condición en la edad adulta. Esto significa que existen varios fenotipos asmáticos:

- Algunos individuos presentan sibilancias transitorias durante la infancia, pero no desarrollarán asma, porque estas sibilancias no se deben a un fenómeno atópico.

- Hay otros, en cambio, que tendrán sibilancias relacionadas con atopia y es probable que desarrollen asma en la etapa adulta.

- Otros enfermos presentarán asma de inicio tardío, después de los cinco años; en 30\% de los casos, ellos serán asmáticos durante la vejez.

- Por último, algunos pacientes desarrollarán asma durante la etapa adulta, de distintos tipos: asma intrínseca, inducida por ácido acetilsalicílico u ocupacional. ${ }^{2}$

En suma, existen varios patrones asmáticos; algunos se van a manifestar durante la infancia, otros durante la adolescencia y otros, incluso, durante la edad adulta, pero la predisposición para ser asmático se comienza a gestar en la vida intrauterina, a medida que dentro del sistema inmunológico fetal se van desarrollando los linfocitos Thelper 2 (Th-2), los que después del nacimiento pueden seguir dos caminos: por uno de ellos, la exposición a factores ambientales o a sarampión, hepatitis o BCG determinará que esta inmunidad tipo 2 varíe a inmunidad tipo 1, que es inocua del punto de vista alérgico; por el otro camino, la exposición a algún factor ambiental durante la infancia dará inicio a un estado inflamatorio de la vía aérea asociado a síntomas asmáticos, estado que se puede resolver durante la infancia o puede continuar hasta la edad adulta y acompañarse de fenómenos de remodelación de la vía aérea y por ende, asma persistente e hiperreactividad bronquial. ${ }^{2}$ 
Si bien no hay una relación directa entre el aumento de asma y la contaminación ambiental, se sabe que los incrementos en los niveles de sulfuro u óxido nitroso se asocian a aumento de las consultas de urgencia de los pacientes asmáticos, que existe una relación entre los altos niveles de óxido nitroso y ozono y la mortalidad del asma y que cuando los niveles de óxido nitroso y algunos peróxidos están elevados, aumenta el uso de broncodilatadores por parte de los pacientes. Otro de los argumentos que se esgrimen para explicar el aumento de la incidencia del asma son los cambios en la dieta, que en occidente se caracteriza por menor consumo de vegetales y productos naturales. En un estudio se encontró que, al disminuir el consumo de estos alimentos, la incidencia de asma aumentó en forma exponencial. ${ }^{2}$

La patogenia del asma desde el punto de vista inmunológico. Existe una susceptibilidad genética, sobre la cual actúan factores ambientales que alteran el medio inmunológico y determinan aumento en la expresión de los linfocitos Th-2, dando origen al estado atópico, en el que la enfermedad asmática se expresa como inflamación, hiperreactividad y obstrucción de la vía aérea. A nivel molecular, el antígeno se une a una célula presentadora, la que estimula a un linfocito $\mathrm{T}$ nativo a nivel de la mucosa respiratoria. Si este linfocito $\mathrm{T}$ nativo monta una respuesta $\mathrm{Th} 2$, un sinnúmero de mediadores químicos, o citoquinas, estimulará la liberación de inmunoglobulina E y de sustancias desde los mastocitos, basófilos y eosinófilos, con lo que se generará un acúmulo de mediadores de inflamación, que causarán hiperreactividad bronquial, obstrucción de la vía aérea y sintomatología asmática. Si el linfocito $\mathrm{T}$ nativo determina una respuesta Th1, se producirá inmunidad celular e inflamación por neutrófilos y no se desencadenará asma. Aún no se sabe por qué un individuo va a la vía Th-2 y no a la vía Th-1. En resumen, el asma bronquial es un desequilibrio entre los mediadores de inflamación (mediadores lipídicos, citoquinas, péptidos, oxidantes) y los mediadores antiinflamatorios (IL-10, IL1RA, INF G, IL-12, 1L-18, PGE 2), a favor de los primeros. ${ }^{2}$

\section{Factores de Riesgo}

Algunos individuos están predispuestos al asma que se convierte con antecedentes familiares fuertes de la enfermedad. La Exposición a las substancias y a las partículas inhaladas que provocan una irritación o una reacción alérgica en las aerovías es un factor de riesgo importante para la enfermedad. Los Irritantes pueden incluir el polvo, el polen, el molde, el humo, la substancia química o la contaminación. ${ }^{7}$ 
Otros disparadores que pueden ascender la presentación de los síntomas del asma incluyen el aire frío, la cólera, el miedo y el ejercicio físico. Además, algunas medicaciones tales como drogas antiinflamatorias no-esteroidales (NSAIDs) y los moldes beta pueden accionar síntomas. ${ }^{7}$

\section{Diagnostico}

Las guías de referencia en el asma ${ }^{8-9}$ establecen que la historia clínica exhaustiva y dirigida, el examen físico, las pruebas de función pulmonar en niños colaboradores y descartar otros diagnósticos son los pasos a seguir para establecer el diagnóstico o la elevada probabilidad de asma. Los aspectos claves para el diagnóstico se detallan en la tabla. ${ }^{10}$

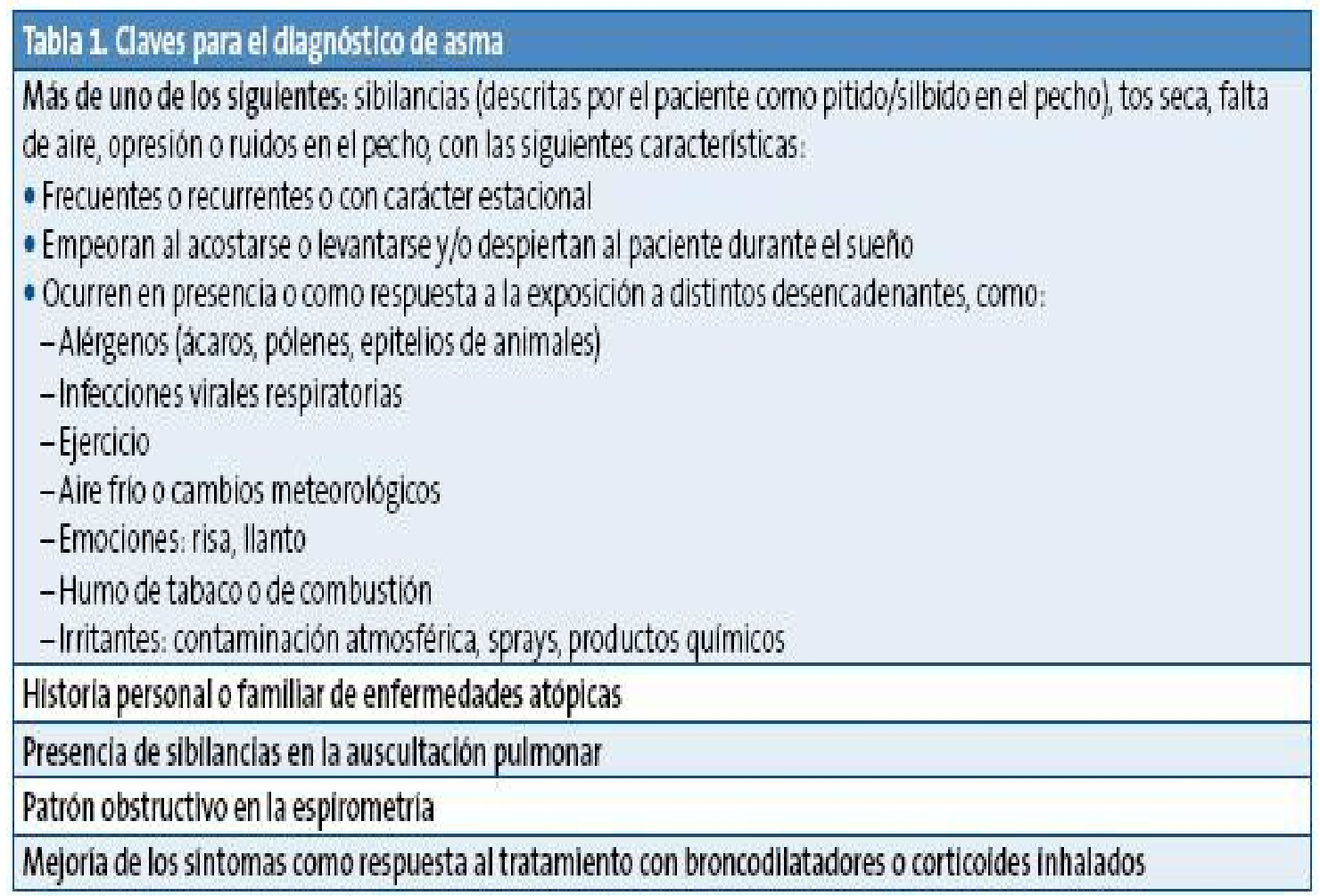

Modificada de: British Guideline on the Management of Asthma (BT5, 2012).

El objetivo del diagnóstico y el tratamiento precoces es limitar la frecuencia y gravedad de las exacerbaciones, impedir el deterioro de la función pulmonar, prevenir la muerte por asma y mantener la calidad de vida del niño o adolescente y su familia. ${ }^{11-10}$ 


\section{Síntomas}

El asma se diagnostica a partir de los datos clínicos, sin embargo, ningún síntoma ni signo clínico es patognomónico y los más frecuentes, la tos y el pitido o silbido en el pecho, son muy poco sensibles. Aparecen habitualmente con un patrón recurrente característico, relacionado con la exposición a desencadenantes reconocibles, y mejoran o revierten con el tratamiento broncodilatador, aunque la falta de respuesta no excluye el diagnóstico. En otros pacientes los síntomas son continuos o persistentes ${ }^{10}$.

\section{Exploración física}

La auscultación pulmonar es con frecuencia normal fuera de los episodios agudos. Las sibilancias son el signo más característico y su detección sugiere una crisis o mal control de la enfermedad. Se deben buscar signos clínicos de atopia y manifestaciones de enfermedades comórbidas, como la dermatitis atópica y la rinitis alérgica. ${ }^{10}$

\section{Prevención}

En la actualidad no se puede prevenir el asma. Sin embargo, se pueden tomar medidas para controlar la enfermedad y prevenir los síntomas. ${ }^{12}$

Medidas a tener en cuenta: conocimiento acerca de la enfermedad, usar las medicinas prescriptas, identificar y tratar de evitar en la medida de lo posible las cosas que empeoren el asma (los desencadenantes del asma). Sin embargo, un desencadenante que no se debe de evitar es la actividad física. La actividad física es un componente importante de un estilo de vida saludable y por ultimo chequeo periodo al especialista. ${ }^{12}$

Resultado de una investigación realizada en el Hospital Baca Ortiz en la ciudad de Quito, se encontró que la edad con mayor prevalencia de asma fue la comprendida entre 2 años y 5 años 11 meses, dentro de los factores de riesgo fueron las infecciones fueron el principal factor para la exacerbación del asma recibieron tratamiento con agonista selectivoß2 -adrenérgico (salbutamol). 
Berbesí-Fernández et al ${ }^{13}$ luego de una intervención familiar y un año de seguimiento de las familias, no se reportaron hospitalizaciones en los niños, un $56 \%$ no requirió atención en un servicio de urgencias, así como una cuarta parte de los niños no reportaron crisis asmáticas atendidas en casa.

De manera semejante Zambrano-Rivera $\mathrm{M}^{14}$ en su investigación de niños hospitalizados con el diagnóstico de asma bronquial en crisis aguda estudio, resulto un número de niños importante donde no se encontraron complicaciones en su hospitalización, existiendo un pequeño número de ellos con insuficiencia respiratoria aguda y otros menos con atelectasia y desequilibrio ácido-básico. El servicio de respiratorio mayor recibió la mayor cuantía de ingresos: todos los niños sobrevivieron con el tratamiento impuesto; con lo cual se aprecia el seguimiento de estos pacientes asmáticos en crisis.

Reportaron una alta prevalencia de asma y síntomas respiratorios crónicos entre niños en una zona deteriorada de una comunidad de descendiente de africanos en las costas de ecuador. La asociación fue encontrada para síntomas respiratorios y exposición, dentro de los hogares, el hábito de fumar paterno y materno, humo de la madera y cucarachas. El resultado sugirió que el asma y los problemas respiratorio tiene alta prevalencia en esta área del mundo y que la población previamente no investigada comparte los mismos factores de riesgo que han sido detectados en algunos países desarrollados. ${ }^{15}$

Otros autores ${ }^{16}$ demostraron en su investigación que las intervenciones educativas son efectivas para mejorar la salud y la calidad de vida de los pacientes con asma, y para reducir el uso y el costo de recursos sanitarios. Estos hallazgos señalan la importancia de incluir el componente educativo como parte esencial del abordaje integral de esta población clínica. Asimismo, la complejidad inherente al proceso educativo pone de manifiesto la necesidad del trabajo conjunto y complementario entre diferentes profesionales de la salud.

\section{Referencias Bibliográficas}

1- Río-Navarro Blanca Estela del, Hidalgo-Castro Emilia María, Sienra-Monge Juan José Luis. Asma. Bol. Med. Hosp. Infant. Mex. 2009 [citado 2017 sept 06] ; 66( 1 ): 3-33. Disponible en:

http://www.scielo.org.mx/scielo.php?script=sci arttext\&pid=S1665$\underline{11462009000100002 \& \ln g=e s}$. 
2- Jover Lopez E . Asma bronquial I: Epidemiología, Patogenia, Definición. Medwave 2007 . . [citado 2017 sept 06] 7 (1):e1901 doi: 10.5867/medwave.2007.01.1901. Disponible en: https://www.medwave.cl/link.cgi/Medwave/PuestaDia/APS/1901

3- The Global Initiative for Asthma. Global Strategy for Asthma Management and Prevention; 2006.

4 Global Initiative for asthma. Pocket guide for asthma management and prevention (for adults and children alder than 5 years). uptodate; 2015.

5- Lang,J. Obesity, Nutrition, and Asthma in Children. Pediatric Allergy, Immunology, and Pulmonology. 2012.

6- Vistín Pazmiño J V . Asma y factores de riesgo como causa de hospitalización en Hospital Baca Ortiz. Informe final de investigación presentado como requisito para optar por el título de especialista en Pediatría. 2017 [citado 2017 sept 06] Disponible en: www.dspace.uce.edu.ec/bitstream/25000/11139/1/T-UCE-0006-009-2017.pdf

7- Smith, BPharm Y. Epidemiología del Asma. 2016. https://www.newsmedical.net/health/Asthma-Epidemiology-\%28Spanish\%29.aspx

8- -Expert Panel Report 3 (EPR3): Guidelines for the Diagnosis and Management of Asthma 2007 [citado 2017 sept 06]

Disponible en:

\section{www.nhlbi.nih.gov/guidelines/asthma/asthgdln.htm}

9- . Brand PLP, Baraldi E, Bisgaard H, Boner AL, Castro-Rodríguez JA, Custovic A, et al. Definition, assessment and treatment of wheezing disorders in preschool children: an evidence -based approach. ERS Task Force. Eur Respir J. 2008;32:1096-110.

10- García Merino Á., Mora Gandarillas I.. Diagnóstico del asma. Rev Pediatr Aten Primaria . 2013. [citado 2017 sept 06] ; 15( Suppl 23 ): 89-95Disponible en: http://scielo.isciii.es/scielo.php?script=sci_arttext\&pid=S113976322013000300010\&lng=es. http://dx.doi.org/10.4321/S1139-76322013000300010. 
11- Asensi Monzó MT, Castillo Laita JA, Esteller Carceller M. Diagnóstico del asma. El Pediatra de Atención Primaria y el Diagnóstico de Asma. Documentos técnicos del GVR [citado 2017 sept 06]. Disponible en http://aepap.org/grupos/grupo-de-vias-respiratoria

12- Martinez J . Causas, Síntomas y Prevención del asma 2012. [citado 2017 sept 06]. Disponible en https:/www.hospitalvernaza.med.ec/blog/item/701-causas-sintomas-v-prevenciondel-asma

13- Berbesí-Fernández D Y, Segura-Cardona A ,Ggarcía-Jaramillo M, Posada-Saldarriaga R .Seguimiento a una cohorte de niños con asma luego de una intervención familiar. Revista CES MEDICINA 2013. 27(2)

14 Zambrano-Rivera M. Características clínicas epidemiológicas del asma bronquial en niños asmáticos en crisis. Dom. Cien 2016 [citado 2017 sept 06]. 2( 4), pp. 51-59 2016 Disponible en :http://dominiodelasciencias.com/ojs/index.php/es/index

15- Hararí R, Forastiere F, Vaca Rodríguez A, Bernal Torres J , Julio Caicedo Guzmán J , Gaspar Andrade C, Axelson O. Pobreza y otros factores de riesgo para el asma ; y las sibilancias entre niños afroecuatorianos. 2004. [citado 2017 sept 06]. Disponible en https://books.google.com.cu/books?isbn=9978224742

16- Fasciglione M P, Castañeiras C E . El componente educativo en el abordaje integral del asma bronquial. J Bras Pneumol. 2010;36(2):252-259 